\title{
Reliability and Availability of Ship's Computer Systems Based on Manufacturer's Data and Worksheets
}

\section{Pouzdanost i raspoloživost brodskih računalnih sustava na temelju podataka i deklaracija proizvođača}

\author{
Igor Vujović \\ University of Split \\ Faculty of Maritime Studies \\ E-mail: ivujovic@pfst.hr
}

\author{
Ivica Kuzmanić \\ University of Split \\ Faculty of Maritime Studies \\ E-mail: ivica.kuzmanic@pfst.hr
}

\author{
DOI 10.17818/NM/2020/3.11 \\ UDK 004.4:519.217 \\ 629.5 \\ Stručni rad / Professional pape \\ Rukopis primljen / Paper accepted: 9. 11. 2019.
}

\section{KEY WORDS}

computer simulation

Markov mode

system's redundancy

reliability

availability of the computer system, which consists of three components with hot standby. Markov chains model is used to analyse probability of failure. Matrix of transitions is set after model development. Transition matrix is used to develop differential equations for model simulation. System's reliability is larger if the system is under constant maintenance and service, but it would not be available. Hence, the optimum between reliability and availability should be found. System's maintenance is limited during the voyage and hot standby is necessary to ensure success of the voyage. This paper presents a framework for assessment of reliability and availability of computer systems based on components' redundancy, and practical data about MTBF. Many versions of the shipboard computer systems can be evaluated using the presented framework.

\section{Sažetak}

Računalno upravljani sustavi imaju značajnu ulogu na brodovima. Kvar tih sustava zbog neispravnosti neke od sastavnica može imati kobne posljedice. Važno je procijeniti pouzdanost i raspoloživost tih sustava te njihovu minimalnu redundantnost da bi se osiguralo planiranje održavanja, naručivanje rezervnih dijelova i sigurna plovidba, uz što manje redundantnih sastavnica. Ovaj rad bavi se razvojem modela pouzdanosti i raspoloživosti računalnog sustava, koji se sastoji od triju sastavnica sa standby opcijom. Model Markovljevih lanaca koristi se za analizu vjerojatnosti greške. Matrica prijelaza sastavljena je nakon razvijanja modela. Ona se koristi za razvoj diferencijalnih jednadžbi za simulaciju modela. Pouzdanost je sustava veća ako se on neprekidno održava i servisira, ali to ne bi bilo raspoloživo. Stoga bi trebalo naći najbolje rješenje između pouzdanosti i raspoloživosti. Održavanje sustava ograničeno je tijekom plovidbe, a standby neophodan je da bi se osiguralo njezino uspješno obavljanje. U radu predstavlja se okvir za procjenu pouzdanosti i raspoloživosti računalnih sustava koji se temelje na redundantnosti sastavnice te na praktičnim podacima o MTBF-u. S pomoću predstavljenog okvira može se ocijeniti puno različitih inačica brodskih računalnih sustava.

\section{INTRODUCTION / Uvod}

Reliability and availability of all systems in transport and industry play an important role in choosing a product by a customer [1]. This directly influences a planned maintenance [2, 3]. Reliability and availability is of such importance that companies build databases to calculate i.e. MTTR, which is presented in [2]. Such collected data are used to detect faults [3] in ships' systems. Computer systems are incorporated in systems of larger scale as control and/or monitoring parts/units. There are differences between computer systems in their construction by application needs, i.e. industrial computers are quite different from PCs. In this paper, special computer is considered which consist of limited number of components.

An example of reliability and availability research is explained in [1], where authors model a quality control system, which is based on computers. However, influencing parameters are hardware, software, and environment. A core part of any computer system (hardware part) is microprocessor unit. All processor manufacturers invest in ensuring long processor lifetime by limiting failures [4]. Mechanisms for failures are researched world-wide. In [4] wear-out related hard errors are considered. The mechanisms of such failures lay in several phenomena, i.e. stress migration, electromigration or time-dependent dielectric breakdown (TDDB). Processor long-term reliability is usually represented by the Bathtub Curve [5], which consists of three parts - early life, useful life and wear-out. Every part of the curve is characterized by different failure mechanism. Since long-term processor reliability is almost completely dependent on intrinsic failures and wear-out, so-called reliability awareness microarchitectural design (RAMP) model is introduced in [6]. RAMP is interesting due to aggressive transistor scaling and increasing processor power, which leads to increase in temperature and demands more efforts in thermal design of microprocessors. In order to incorporate other parts of computer in the analysis, a framework for architecture-level lifetime reliability modelling is 
introduced in [7]. The research includes Monte Carlo simulations and effective combination of low-level effects and architecturallevel effects. Mechanisms for failure analysed are: electromigration, negative bias temperature instability (NBTI) and TDDB. The work includes other components of the system, such as SRAM (Static Random-Access Memory) and redundant systems.

Reliability of memory was addressed in [8-11]. Reliability of ferroelectric RAM was analysed in [8]. Design of fault-tolerant RAM was the scope of [9]. Optimization criteria were considered in [11]. Criteria were minimization of costs, maximization of equipment availability, and the achievement of a desired stock reliability. Normal distribution and Poisson process approach were used for non-repairable components.

Reliability and availability of an industrial computer system was presented in [1], which is similar to ship's system due to the scope of such systems. The difference is in a fact that ship's system should not fail between two harbours. So, the situations with double failure (main and redundant components) should be avoided.

This paper is organized as follows. In section 2, we describe the process of model development. In section 3, the results of simulation are presented and discussed. Section 4 is the conclusion section.

\section{DEVELOPING SIMULATION MODEL FROM THEORY / Razvoj simulacijskog modela iz teorije}

In order to determine system's availability, it is necessary to develop simplified model and exploit it. Availability is defined by [12]:

$$
A_{i}=\frac{M T B F}{M T B F+M T T R}
$$

where MTTR is Mean Time To Repair and MTBF Mean Time Before Failure. Availability is often expressed as:

$$
A_{i}=\frac{\mu}{\mu+\lambda}
$$

where $\lambda$ is the intensity of failures and $\mu$ the intensity of repairs. Intensity of failures can be determined by $[12,13]$ :

$$
\lambda=\frac{1}{M T B F}
$$

Intensity of repairs is defined with [12]:

$$
\mu=\frac{1}{M T T R}
$$

Simulation model considered deals only with hardware part of the computer system. In order to simplify the model, only three crucial components were taken into account. The research can be extended for more components if necessary for some specific purpose. Every component have hot standby in parallel branch. Considered components of the computer system are: microprocessor (MP), random access memory (RM) and hard disk (TD). Considered computer system can be shown by block-diagram in Figure 1. Redundant components are shown in parallel branches. Since, computer fails if any of parallel branches fails, three parallel branches are connected into series (see reliability in parallel [13]). There are several more components that could be taken account, but necessary data have not been available at time of research. Hence, this is a simplified model.

Table 1 shows all possible states of the system. However, it is necessary to consider only situations which do not lead to system failure. Operational component is in state " 0 " and the component is state of failure is in state " 1 ". For example, state 1 can be expressed as: $M P 1 \& M P 2 \& R M 1 \& R M 2 \& T D 1 \& T D 2$. If some component is in state of failure, then it is written with negation (i.e. $\overline{T D 2}$ ).

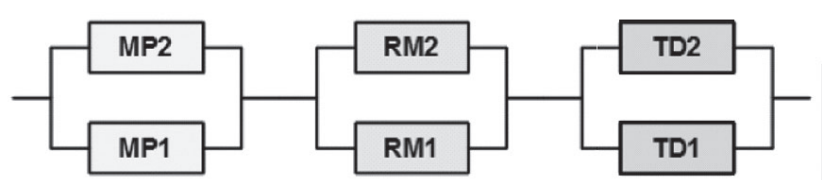

Figure 1 Considered system Slika 1. Razmatrani sustav

All allowed cases (27 in total) can be reduced by different formulation. Cases when one component in parallel is operational andoneinfailurestatecanbeexpressedasonenewcasewhenparallel are operational. For example, cases no 2 and 3 from Table 1 can be

\begin{tabular}{|c|c|c|c|c|c|c|}
\hline No. & MP1 & MP2 & RM1 & RM2 & TD1 & TD2 \\
\hline 1 & 0 & 0 & 0 & 0 & 0 & 0 \\
\hline 2 & 0 & 0 & 0 & 0 & 0 & 1 \\
\hline 3 & 0 & 0 & 0 & 0 & 1 & 0 \\
\hline 4 & $\theta$ & $\theta$ & $\theta$ & $\theta$ & 7 & $\neq$ \\
\hline 5 & 0 & 0 & 0 & 1 & 0 & 0 \\
\hline 6 & 0 & 0 & 0 & 1 & 0 & 1 \\
\hline 7 & 0 & 0 & 0 & 1 & 1 & 0 \\
\hline 8 & $\theta$ & $\theta$ & $\theta$ & $\neq$ & 7 & 7 \\
\hline 9 & 0 & 0 & 1 & 0 & 0 & 0 \\
\hline 10 & 0 & 0 & 1 & 0 & 0 & 1 \\
\hline 11 & 0 & 0 & 1 & 0 & 1 & 0 \\
\hline 12 & $\theta$ & $\theta$ & $\neq$ & $\theta$ & $\neq$ & $\neq$ \\
\hline 13 & $\theta$ & $\theta$ & 7 & 7 & $\theta$ & $\theta$ \\
\hline 14 & $\theta$ & $\theta$ & 7 & 7 & $\theta$ & 7 \\
\hline 15 & $\theta$ & $\theta$ & 7 & 7 & 7 & $\theta$ \\
\hline 16 & $\theta$ & $\theta$ & 7 & 7 & 7 & 7 \\
\hline 17 & 0 & 1 & 0 & 0 & 0 & 0 \\
\hline 18 & 0 & 1 & 0 & 0 & 0 & 1 \\
\hline 19 & 0 & 1 & 0 & 0 & 1 & 0 \\
\hline 20 & $\theta$ & $\neq$ & $\theta$ & $\theta$ & 7 & $\neq$ \\
\hline 21 & 0 & 1 & 0 & 1 & 0 & 0 \\
\hline 22 & 0 & 1 & 0 & 1 & 0 & 1 \\
\hline 23 & 0 & 1 & 0 & 1 & 1 & 0 \\
\hline 24 & $\theta$ & 7 & $\theta$ & 7 & 7 & 7 \\
\hline 25 & 0 & 1 & 1 & 0 & 0 & 0 \\
\hline 26 & 0 & 1 & 1 & 0 & 0 & 1 \\
\hline 27 & 0 & 1 & 1 & 0 & 1 & 0 \\
\hline 28 & $\theta$ & 7 & 7 & $\theta$ & 7 & 7 \\
\hline 29 & $\theta$ & 7 & 7 & 7 & $\theta$ & $\theta$ \\
\hline 30 & $\theta$ & $\neq$ & 7 & z & $\theta$ & z \\
\hline 31 & $\theta$ & 7 & 7 & 7 & 7 & $\theta$ \\
\hline 32 & $\theta$ & 7 & 7 & $\neq$ & 7 & 7 \\
\hline 33 & 1 & 0 & 0 & 0 & 0 & 0 \\
\hline 34 & 1 & 0 & 0 & 0 & 0 & 1 \\
\hline 35 & 1 & 0 & 0 & 0 & 1 & 0 \\
\hline 36 & 7 & $\theta$ & $\theta$ & $\theta$ & 7 & 7 \\
\hline 37 & 1 & 0 & 0 & 1 & 0 & 0 \\
\hline 38 & 1 & 0 & 0 & 1 & 0 & 1 \\
\hline 39 & 1 & 0 & 0 & 1 & 1 & 0 \\
\hline 40 & 7 & $\theta$ & $\theta$ & $\neq$ & 7 & $\neq$ \\
\hline 41 & 1 & 0 & 1 & 0 & 0 & 0 \\
\hline 42 & 1 & 0 & 1 & 0 & 0 & 1 \\
\hline 43 & 1 & 0 & 1 & 0 & 1 & 0 \\
\hline 44 & 7 & $\theta$ & $\neq$ & $\theta$ & 7 & $\neq$ \\
\hline 45 & 7 & $\theta$ & 7 & 7 & $\theta$ & $\theta$ \\
\hline 46 & $\neq$ & $\theta$ & 7 & 7 & $\theta$ & $\neq$ \\
\hline 47 & 7 & $\theta$ & 7 & 7 & 7 & $\theta$ \\
\hline 48 & 7 & $\theta$ & 7 & 7 & 7 & 7 \\
\hline 49 & 7 & $\neq$ & $\theta$ & $\theta$ & $\theta$ & $\theta$ \\
\hline 50 & 7 & 7 & $\theta$ & $\theta$ & $\theta$ & 7 \\
\hline 51 & $\neq$ & $\neq$ & $\theta$ & $\theta$ & $\neq$ & $\theta$ \\
\hline 52 & $\neq$ & $\neq$ & $\theta$ & $\theta$ & 7 & 7 \\
\hline 53 & 7 & 7 & $\theta$ & 7 & $\theta$ & $\theta$ \\
\hline 54 & 7 & 7 & $\theta$ & 7 & $\theta$ & 7 \\
\hline 55 & $\neq$ & $\neq$ & $\theta$ & 7 & $\neq$ & $\theta$ \\
\hline 56 & $\neq$ & $\neq$ & $\theta$ & 7 & $\neq$ & $\neq$ \\
\hline 57 & $\neq$ & $\neq$ & $\neq$ & $\theta$ & $\theta$ & $\theta$ \\
\hline 58 & 7 & 7 & 7 & $\theta$ & $\theta$ & 7 \\
\hline 59 & $\neq$ & $\neq$ & $\neq$ & $\theta$ & $\neq$ & $\theta$ \\
\hline 60 & 7 & 7 & 7 & $\theta$ & 7 & 7 \\
\hline 61 & $\neq$ & $\neq$ & $\neq$ & $f$ & $\theta$ & $\theta$ \\
\hline 62 & $\neq$ & $\neq$ & 7 & 7 & $\theta$ & $\neq$ \\
\hline 63 & $\neq$ & $\neq$ & 7 & $q$ & $\neq$ & $\theta$ \\
\hline 64 & 7 & 7 & $\neq$ & $\neq$ & $\neq$ & $\neq$ \\
\hline
\end{tabular}
written as one case: $M P 1 * M P 2 * R M 1 * \mathrm{RM} 2 *(\overline{T D 1}+\overline{T D 2})$.

Table 1 Possible states of the system (not double strikethrough) Tablica 1. Moguća stanja sustava (ne dvaput precrtano) 
Therefore, 27 states can be reduced to just 9 states with 7 transitions. State $\mathrm{S} 0$ is defined as the state with all components operational. If one component in parallel fails, transition from S0 to S1 (for MP failure), S2 (for RM failure) or S3 occurs (for TD failure). System cannot return from states S1, S2 and S3 to S0, because there are no repairs intensities. If the system is not maintained (repaired), the performance can be even worse. From S1, system can degrade to S4 or S5 or in total failure SK (state of failure). From S2, system can change its condition to states S4, S6 or SK. From S3, system can deteriorate to S5, S6 or SK. From new states, S4, S5 or S6, system can degrade to S7 or SK, and, finally, from S7 only to SK. Table 2 shows reduced states.

Table 2 Reduced system states

Tablica 2. Reducirana stanja sustava

\begin{tabular}{|c|c|}
\hline State & State's description \\
\hline $\mathrm{S}_{0}$ & $M P 1 \& M P 2 \& R M 1 \& R M 2 \& T D 1 \& T D 2$ \\
\hline $\mathrm{S}_{1}$ & $(\overline{M P 1} \mid \overline{M P 2}) \& R M 1 \& R M 2 \& T D 1 \& T D 2$ \\
\hline $\mathrm{S}_{2}$ & $M P 1 \& M P 2 \&(\overline{R M 1} \mid \overline{R M 2}) \& T D 1 \& T D 2$ \\
\hline $\mathrm{S}_{3}$ & $M P 1 \& M P 2 \& R M 1 \& R M 2 \&(\overline{T D 1} \mid \overline{T D 2})$ \\
\hline $\mathrm{S}_{4}$ & $(\overline{M P 1} \mid \overline{M P 2}) \&(\overline{R M 1} \mid \overline{R M 2}) \& T D 1 \& T D 2$ \\
\hline $\mathrm{S}_{5}$ & $(\overline{M P 1} \mid \overline{M P 2}) \& R M 1 \& R M 2 \&(\overline{T D 1} \mid \overline{T D 2})$ \\
\hline $\mathrm{S}_{6}$ & $M P 1 \& M P 2 \&(\overline{R M 1} \mid \overline{R M 2}) \&(\overline{T D 1} \mid \overline{T D 2})$ \\
\hline $\mathrm{S}_{7}$ & $(\overline{M P 1} \mid \overline{M P 2}) \&(\overline{R M 1} \mid \overline{R M 2}) \&(\overline{T D 1} \mid \overline{T D 2})$ \\
\hline
\end{tabular}

Previous description can be shown in graphical representation as Markov model [14] of system reliability, as in Figure 2. Probabilities of state's transition is calculated by introducing failure intensity, $\lambda$, and time interval, $\Delta$ t. State of system failure, SK, occurs where serial connection is interrupted in any part of chain.

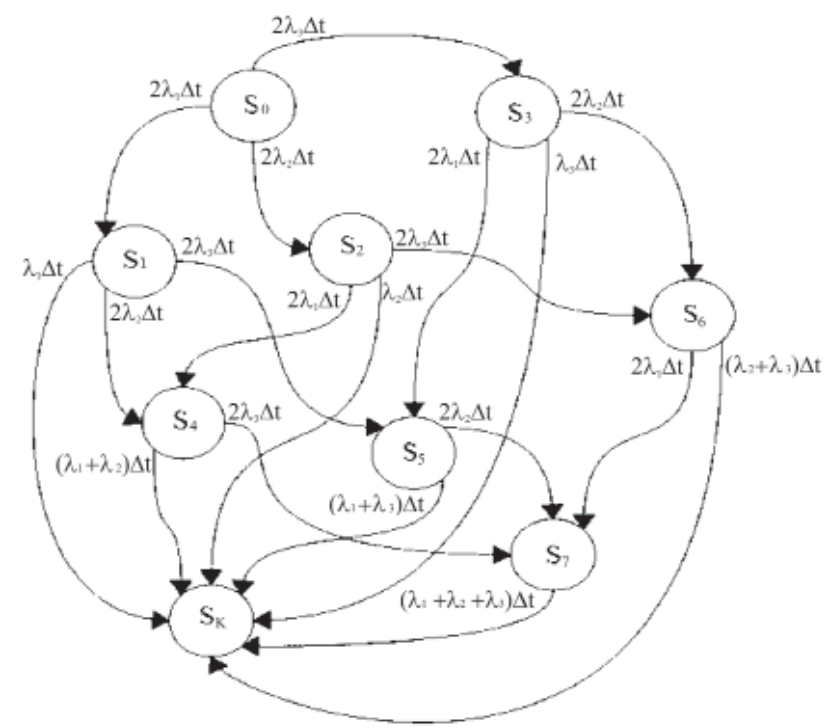

Figure 2 Graphical representation of the Markov model for system reliability

Slika 2. Grafički prikaz Markovljeva modela pouzdanosti sustava

When considering availability of the system, the system can return to previous state. Therefore, one can say that there is a relationship between previous states and new states through intensity of repairs. However, if the system reached the state of failure, it cannot return to the previous state. In order to simulate the system, it is important to get the system's equations. Table 3 shows probabilities of stats' transitions for system's reliability analysis.

Table 3 Transition of states for system's reliability Tablica 3. Prijelaz stanja za pouzdanost sustava

\begin{tabular}{|c|c|c|c|c|c|c|c|c|c|}
\hline \multirow{2}{*}{$P_{S}(t)$} & \multicolumn{9}{|c|}{$P_{S}(t+\Delta t)$} \\
\hline & $P_{50}$ & $P_{s 1}$ & $P_{\mathrm{s} 2}$ & $P_{53}$ & $\mathrm{P}_{54}$ & $P_{55}$ & $P_{56}$ & $P_{57}$ & $P_{S K}$ \\
\hline$P_{50}$ & $\begin{array}{c}1-2\left(\lambda_{1}+\right. \\
\left.\lambda_{2}+\lambda_{3}\right)\end{array}$ & 0 & 0 & 0 & 0 & 0 & 0 & 0 & 0 \\
\hline$P_{s 1}$ & $2 \lambda_{1}$ & $\begin{array}{c}1-\left(\lambda_{1}+\right. \\
\left.2 \lambda_{2}+2 \lambda_{3}\right)\end{array}$ & 0 & 0 & 0 & 0 & 0 & 0 & 0 \\
\hline$P_{s 2}$ & $2 \lambda_{2}$ & 0 & $\begin{array}{l}1-\left(2 \lambda_{1}+\right. \\
\left.\lambda_{2}+2 \lambda_{3}\right)\end{array}$ & 0 & 0 & 0 & 0 & 0 & 0 \\
\hline$P_{53}$ & $2 \lambda_{3}$ & 0 & 0 & $\begin{array}{l}1-\left(2 \lambda_{1}+\right. \\
\left.2 \lambda_{2}+\lambda_{3}\right)\end{array}$ & 0 & 0 & 0 & 0 & 0 \\
\hline$P_{54}$ & 0 & $2 \lambda_{2}$ & $2 \lambda_{1}$ & 0 & $\begin{array}{c}1-\left(\lambda_{1}+\right. \\
\left.\lambda_{2}+2 \lambda_{3}\right)\end{array}$ & 0 & 0 & 0 & 0 \\
\hline$P_{55}$ & 0 & $2 \lambda_{3}$ & 0 & $2 \lambda_{1}$ & 0 & $\begin{array}{c}1-\left(\lambda_{1}+\right. \\
\left.2 \lambda_{2}+\lambda_{3}\right)\end{array}$ & 0 & 0 & 0 \\
\hline$P_{56}$ & 0 & 0 & $2 \lambda_{3}$ & $2 \lambda_{2}$ & 0 & 0 & $\begin{array}{l}1-\left(2 \lambda_{1}+\right. \\
\left.\lambda_{2}+\lambda_{3}\right)\end{array}$ & 0 & 0 \\
\hline$P_{57}$ & 0 & 0 & 0 & 0 & $2 \lambda_{3}$ & $2 \lambda_{2}$ & $2 \lambda_{1}$ & $1-\left(\lambda_{1}+\lambda_{2}+\lambda_{3}\right)$ & 0 \\
\hline$P_{S K}$ & 0 & $\lambda_{1}$ & $\lambda_{2}$ & $\lambda_{3}$ & $\lambda_{1}+\lambda_{2}$ & $\lambda_{1}+\lambda_{3}$ & $\lambda_{2}+\lambda_{3}$ & $\lambda_{1}+\lambda_{2}+\lambda_{3}$ & 1 \\
\hline
\end{tabular}


From Table 3, it is possible to write system's states as (5) - (13):

$$
\begin{gathered}
P_{S 0}(t+\Delta t)=P_{S 0}(t)\left[1-2\left(\lambda_{1}+\lambda_{2}+\lambda_{3}\right) \Delta t\right] \\
P_{S I}(t+\Delta t)=P_{S I}(t)\left[1-\left(\lambda_{1}+2 \lambda_{2}+2 \lambda_{3}\right) \Delta t\right]+P_{S 0}(t) 2 \lambda_{1} \Delta t \\
P_{S 2}(t+\Delta t)=P_{S 2}(t)\left[1-\left(2 \lambda_{1}+\lambda_{2}+2 \lambda_{3}\right) \Delta t\right]+P_{S 0}(t) 2 \lambda_{2} \Delta t \\
P_{S 3}(t+\Delta t)=P_{S 3}(t)\left[1-\left(2 \lambda_{1}+2 \lambda_{2}+\lambda_{3}\right) \Delta t\right]+P_{S 0}(t) 2 \lambda_{3} \Delta t \\
P_{S 4}(t+\Delta t)=P_{S 4}(t)\left[1-\left(\lambda_{1}+\lambda_{2}+2 \lambda_{3}\right) \Delta t\right]+P_{S I}(t) 2 \lambda_{2} \Delta t+P_{S 2}(t) 2 \lambda_{1} \Delta t \\
P_{S 5}(t+\Delta t)=P_{S 5}(t)\left[1-\left(\lambda_{1}+2 \lambda_{2}+\lambda_{3}\right) \Delta t\right]+P_{S 1}(t) 2 \lambda_{3} \Delta t+P_{S 3}(t) 2 \lambda_{1} \Delta t \\
P_{S 6}(t+\Delta t)=P_{S 6}(t)\left[1-\left(2 \lambda_{1}+\lambda_{2}+\lambda_{3}\right) \Delta t\right]+P_{S 2}(t) 2 \lambda_{3} \Delta t+P_{S 3}(t) 2 \lambda_{2} \Delta t \\
P_{S 7}(t+\Delta t)=P_{S 7}(t)\left[1-\left(\lambda_{1}+\lambda_{2}+\lambda_{3}\right) \Delta t\right]+P_{S 4}(t) 2 \lambda_{3} \Delta t+P_{S 5}(t) 2 \lambda_{2} \Delta t+P_{S 6}(t) 2 \lambda_{1} \Delta t \\
P_{S K}(t+\Delta t)=P_{S K}(t)+P_{S l}(t) \lambda_{1} \Delta t+P_{S 2}(t) \lambda_{2} \Delta t+P_{S 3}(t) \lambda_{3} \Delta t+P_{S 4}(t)\left(\lambda_{1}+\lambda_{2}\right) \Delta t \\
+P_{S 5}(t)\left(\lambda_{1}+\lambda_{3}\right) \Delta t+P_{S 6}(t)\left(\lambda_{2}+\lambda_{3}\right) \Delta t+P_{S 7}(t)\left(\lambda_{1}+\lambda_{2}+\lambda_{3}\right) \Delta t
\end{gathered}
$$

And, after including time-continuity in the equations, the system's states can be transformed to (14) - (22):

$$
\begin{gathered}
\frac{d P_{S 0}}{d t}=P_{S 0}(t)\left[-2\left(\lambda_{1}+\lambda_{2}+\lambda_{3}\right)\right] \\
\frac{d P_{S 1}}{d t}=P_{S 1}(t)\left[-\left(\lambda_{1}+2 \lambda_{2}+2 \lambda_{3}\right)\right]+P_{S 0}(t) 2 \lambda_{1} \\
\frac{d P_{S 2}}{d t}=P_{S 2}(t)\left[-\left(2 \lambda_{1}+\lambda_{2}+2 \lambda_{3}\right)\right]+P_{S 0}(t) 2 \lambda_{2} \\
\frac{d P_{S 3}}{d t}=P_{S 3}(t)\left[-\left(2 \lambda_{1}+2 \lambda_{2}+\lambda_{3}\right)\right]+P_{S 0}(t) 2 \lambda_{3} \\
\frac{d P_{S 4}}{d t}=P_{S 4}(t)\left[-\left(\lambda_{1}+\lambda_{2}+2 \lambda_{3}\right)\right]+P_{S 1}(t) 2 \lambda_{2}+P_{S 2}(t) 2 \lambda_{1} \\
\frac{d P_{S 5}}{d t}=P_{S 5}(t)\left[-\left(\lambda_{1}+2 \lambda_{2}+\lambda_{3}\right)\right]+P_{S 1}(t) 2 \lambda_{3}+P_{S 3}(t) 2 \lambda_{1} \\
\frac{d P_{S 6}}{d t}=P_{S 6}(t)\left[-\left(2 \lambda_{1}+\lambda_{2}+\lambda_{3}\right)\right]+P_{S 2}(t) 2 \lambda_{3}+P_{S 3}(t) 2 \lambda_{2} \\
\frac{d P_{S 7}}{d t}=P_{S 7}(t)\left[-\left(\lambda_{1}+\lambda_{2}+\lambda_{3}\right)\right]+P_{S 4}(t) 2 \lambda_{3}+P_{S 5}(t) 2 \lambda_{2}+P_{S 6}(t) 2 \lambda_{1} \\
\frac{d P_{S K}}{d t}=P_{S 1}(t) \lambda_{1}+P_{S 2}(t) \lambda_{2}+P_{S 3}(t) \lambda_{3}+P_{S 4}(t)\left(\lambda_{1}+\lambda_{2}\right)+P_{S 5}(t)\left(\lambda_{1}+\lambda_{3}\right) \\
+P_{S 6}(t)\left(\lambda_{2}+\lambda_{3}\right)+P_{S 7}(t)\left(\lambda_{1}+\lambda_{2}+\lambda_{3}\right)
\end{gathered}
$$

These equations can be used in simulation model for digital computers (i.e. in Simulink ${ }^{\mathrm{T} M}$ ). By analogy, Table 4 shows transitions of states in system's availability and Figure 3 model of system's availability. From Table 4, it is possible to write system's states as (23) - (31):

$$
\begin{gathered}
P_{S 0}(t+\Delta t)=P_{S 0}(t)\left[1-2\left(\lambda_{1}+\lambda_{2}+\lambda_{3}\right) \Delta t\right]+P_{S 1}(t) \mu_{1} \Delta t+P_{S 2}(t) \mu_{2} \Delta t+P_{S 3}(t) \mu_{3} \Delta t \\
P_{S 1}(t+\Delta t)=P_{S 1}(t)\left[1-\left(\lambda_{1}+2 \lambda_{2}+2 \lambda_{3}\right) \Delta t-\mu_{1} \Delta t\right]+P_{S 0}(t) 2 \lambda_{1} \Delta t+P_{S 4}(t) \mu_{2} \Delta t+P_{S 5}(t) \mu_{3} \Delta t \\
P_{S 2}(t+\Delta t)=P_{S 2}(t)\left[1-\left(2 \lambda_{1}+\lambda_{2}+2 \lambda_{3}\right) \Delta t-\mu_{2} \Delta t\right]+P_{S 0}(t) 2 \lambda_{2} \Delta t+P_{S 4}(t) \mu_{1} \Delta t+P_{S 6}(t) \mu_{3} \Delta t \\
P_{S 3}(t+\Delta t)=P_{S 3}(t)\left[1-\left(2 \lambda_{1}+2 \lambda_{2}+\lambda_{3}\right) \Delta t-\mu_{3} \Delta t\right]+P_{S 0}(t) 2 \lambda_{3} \Delta t+P_{S 5}(t) \mu_{1} \Delta t+P_{S 6}(t) \mu_{2} \Delta t \\
P_{S 4}(t+\Delta t)=P_{S 4}(t)\left[1-\left(\lambda_{1}+\lambda_{2}+2 \lambda_{3}\right) \Delta t-\left(\mu_{1}+\mu_{2}\right) \Delta t\right]+P_{S 1}(t) 2 \lambda_{2} \Delta t+P_{7}(t) \mu_{3} \Delta t \\
P_{S 5}(t+\Delta t)=P_{S 5}(t)\left[1-\left(\lambda_{1}+2 \lambda_{2}+\lambda_{3}\right) \Delta t-\left(\mu_{1}+\mu_{3}\right) \Delta t\right]+P_{S I}(t) 2 \lambda_{3} \Delta t+ \\
+P_{S 3}(t) 2 \lambda_{1} \Delta t+P_{S 7}(t) \mu_{2} \Delta t \\
P_{S 6}(t+\Delta t)=P_{S 6}(t)\left[1-\left(2 \lambda_{1}+\lambda_{2}+\lambda_{3}\right) \Delta t-\left(\mu_{2}+\mu_{3}\right) \Delta t\right]+P_{S 2}(t) 2 \lambda_{3} \Delta t+ \\
+P_{S 3}(t) 2 \lambda_{2} \Delta t+P_{S 7}(t) \mu_{1} \Delta t \\
P_{S 7}(t+\Delta t)=P_{S 7}(t)\left[1-\left(\lambda_{1}+\lambda_{2}+\lambda_{3}\right) \Delta t-\left(\mu_{1}+\mu_{2}+\mu_{3}\right) \Delta t\right]+P_{S 4}(t) 2 \lambda_{3} \Delta t+ \\
P_{S 5}(t) 2 \lambda_{2} \Delta t+P_{S 6}(t) 2 \lambda_{1} \Delta t \\
P_{S K}(t+\Delta t)=P_{S K}(t)+P_{S I}(t) \lambda_{1} \Delta t+P_{S 2}(t) \lambda_{2} \Delta t+P_{S 3}(t) \lambda_{3} \Delta t+P_{S 4}(t)\left(\lambda_{1}+\lambda_{2}\right) \Delta t \\
+P_{S 5}(t)\left(\lambda_{1}+\lambda_{3}\right) \Delta t+P_{S 6}(t)\left(\lambda_{2}+\lambda_{3}\right) \Delta t+P_{S 7}(t)\left(\lambda_{1}+\lambda_{2}+\lambda_{3}\right) \Delta t
\end{gathered}
$$


Table 4 State's transitions for system's availability Tablica 4. Prijelazi stanja za raspoloživost sustava

\begin{tabular}{|c|c|c|c|c|c|c|c|c|c|}
\hline \multirow{2}{*}{$P_{S}(t)$} & \multicolumn{9}{|c|}{$\mathrm{P}_{\mathrm{s}}(\mathrm{t}+\Delta \mathrm{t})$} \\
\hline & $\mathrm{P}_{\text {so }}$ & $P_{s 1}$ & $P_{52}$ & $P_{53}$ & $P_{54}$ & $P_{55}$ & $P_{56}$ & $P_{57}$ & $\mathrm{P}_{\mathrm{SK}}$ \\
\hline$P_{s 0}$ & $1-2\left(\lambda_{1}+\lambda_{2}+\lambda_{3}\right)$ & $\mu_{1}$ & $\mu_{2}$ & $\mu_{3}$ & 0 & 0 & 0 & 0 & 0 \\
\hline$P_{s 1}$ & $2 \lambda_{1}$ & $1-\left(\lambda_{1}+2 \lambda_{2}+2 \lambda_{3}\right)-\mu_{1}$ & 0 & 0 & $\mu_{2}$ & $\mu_{3}$ & 0 & 0 & 0 \\
\hline$P_{s 2}$ & $2 \lambda_{2}$ & 0 & $1-\left(2 \lambda_{1}+\lambda_{2}+2 \lambda_{3}\right)-\mu_{2}$ & 0 & $\mu_{1}$ & 0 & $\mu_{3}$ & 0 & 0 \\
\hline $\mathrm{P}_{\mathrm{S} 3}$ & $2 \lambda_{3}$ & 0 & 0 & $\begin{array}{c}1-\left(2 \lambda_{1}+\right. \\
\left.2 \lambda_{2}+\lambda_{3}\right)-\mu_{3}\end{array}$ & 0 & $\mu_{1}$ & $\mu_{2}$ & 0 & 0 \\
\hline$P_{S 4}$ & 0 & $2 \lambda_{2}$ & $2 \lambda_{1}$ & 0 & $\begin{array}{c}1-\left(\lambda_{1}+\lambda_{2}+2 \lambda_{3}\right) \\
-\left(\mu_{1}+\mu_{2}\right)\end{array}$ & 0 & 0 & $\mu_{3}$ & 0 \\
\hline$P_{55}$ & 0 & $2 \lambda_{3}$ & 0 & $2 \lambda_{1}$ & 0 & $\begin{array}{c}1-\left(\lambda_{1}+2 \lambda_{2}+\lambda_{3}\right)- \\
\left(\mu_{1}+\mu_{3}\right)\end{array}$ & 0 & $\mu_{2}$ & 0 \\
\hline$P_{56}$ & 0 & 0 & $2 \lambda_{3}$ & $2 \lambda_{2}$ & 0 & 0 & $\begin{array}{c}1-\left(2 \lambda_{1}+\lambda_{2}+\lambda_{3}\right) \\
-\left(\mu_{2}+\mu_{3}\right)\end{array}$ & $\mu_{1}$ & 0 \\
\hline$P_{57}$ & 0 & 0 & 0 & 0 & $2 \lambda_{3}$ & $2 \lambda_{2}$ & $2 \lambda_{1}$ & $\begin{array}{c}1-\left(\lambda_{1}+\lambda_{2}+\lambda_{3}\right)- \\
\left(\mu_{1}+\mu_{2}+\mu_{3}\right)\end{array}$ & 0 \\
\hline$P_{\text {SK }}$ & 0 & $\lambda_{1}$ & $\lambda_{2}$ & $\lambda_{3}$ & $\lambda_{1}+\lambda_{2}$ & $\lambda_{1}+\lambda_{3}$ & $\lambda_{2}+\lambda_{3}$ & $\lambda_{1}+\lambda_{2}+\lambda_{3}$ & 1 \\
\hline
\end{tabular}

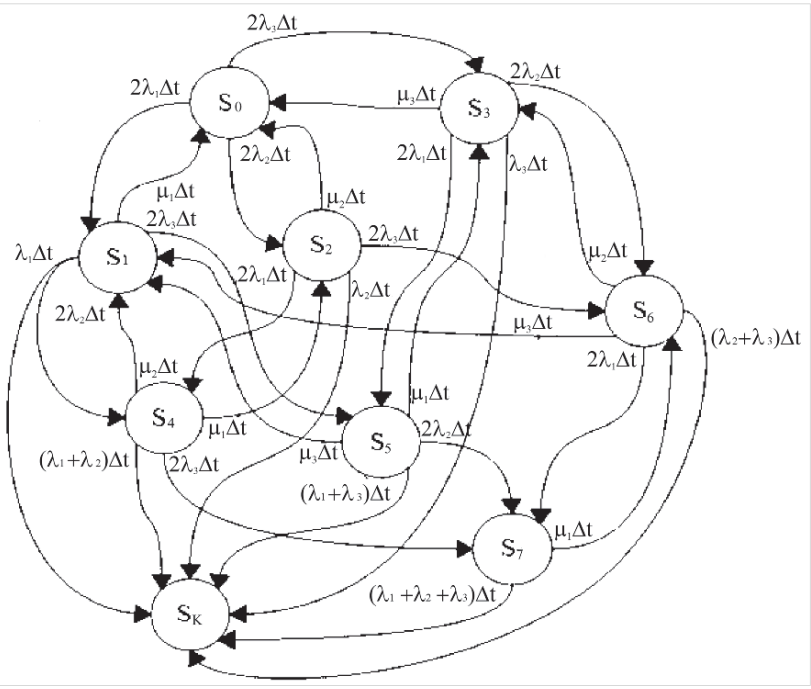

Figure 3 System's availability Slika 3. Raspoloživost sustava

After including time-continuity in the equation, we obtain (32)-(40):

$$
\begin{gathered}
\frac{d P_{S 0}}{d t}=P_{S 0}(t)\left[-2\left(\lambda_{1}+\lambda_{2}+\lambda_{3}\right)\right]+P_{S 1}(t) \mu_{1}+P_{S 2}(t) \mu_{2}+P_{S 3}(t) \mu_{3} \\
\frac{d P_{S 1}}{d t}=P_{S I}(t)\left[-\left(\lambda_{1}+2 \lambda_{2}+2 \lambda_{3}\right)-\mu_{1}\right]+P_{S 0}(t) 2 \lambda_{1}+P_{S 4}(t) \mu_{2}+P_{S 5}(t) \mu_{3} \\
\frac{d P_{S 2}}{d t}=P_{S 2}(t)\left[-\left(2 \lambda_{1}+\lambda_{2}+2 \lambda_{3}\right)-\mu_{2}\right]+P_{S 0}(t) 2 \lambda_{2}+P_{S 4}(t) \mu_{1}+P_{S 6}(t) \mu_{3} \\
\frac{d P_{S 3}}{d t}=P_{S 3}(t)\left[-\left(2 \lambda_{1}+2 \lambda_{2}+\lambda_{3}\right)-\mu_{3}\right]+P_{S 0}(t) 2 \lambda_{3}+P_{S 5}(t) \mu_{1}+P_{S 6}(t) \mu_{2} \\
\frac{d P_{S 4}}{d t}=P_{S 4}(t)\left[-\left(\lambda_{1}+\lambda_{2}+2 \lambda_{3}\right)-\left(\mu_{1}+\mu_{2}\right)\right]+P_{S 1}(t) 2 \lambda_{2}+P_{S 2}(t) 2 \lambda_{1}+P_{S 7}(t) \mu_{3} \\
\frac{d P_{S 5}}{d t}=P_{S 5}(t)\left[-\left(\lambda_{1}+2 \lambda_{2}+\lambda_{3}\right)-\left(\mu_{1}+\mu_{3}\right)\right]+P_{S 1}(t) 2 \lambda_{3}+P_{S 3}(t) 2 \lambda_{1}+P_{S 7}(t) \mu_{2} \\
\frac{d P_{S 6}}{d t}=P_{S 6}(t)\left[-\left(2 \lambda_{1}+\lambda_{2}+\lambda_{3}\right)-\left(\mu_{2}+\mu_{3}\right)\right]+P_{S 2}(t) 2 \lambda_{3}+P_{S 3}(t) 2 \lambda_{2}+P_{S 7}(t) \mu_{1}
\end{gathered}
$$




$$
\begin{gathered}
\frac{d P_{S 7}}{d t}=P_{S 7}(t)\left[-\left(\lambda_{1}+\lambda_{2}+\lambda_{3}\right)-\left(\mu_{1}+\mu_{2}+\mu_{3}\right)\right]+P_{S 4}(t) 2 \lambda_{3}+P_{S 5}(t) 2 \lambda_{2}+P_{S 6}(t) 2 \lambda_{1} \\
\frac{d P_{S K}}{d t}=P_{S 1}(t) \lambda_{1} \\
+P_{S 2}(t) \lambda_{2}+P_{S 3}(t) \lambda_{3}+P_{S 4}(t)\left(\lambda_{1}+\lambda_{2}\right)+P_{S 5}(t)\left(\lambda_{1}+\lambda_{3}\right) \\
+P_{S 6}(t)\left(\lambda_{2}+\lambda_{3}\right)+P_{S 7}(t)\left(\lambda_{1}+\lambda_{2}+\lambda_{3}\right)
\end{gathered}
$$

These equations were used in building the actual simulation model in Simulink ${ }^{\mathrm{TM}}$.

\section{RESULTS / Rezultati \\ 3.1. Setup / Plan rada}

Firstly, reliability of the considered system is simulated. In the first step, simulation model is used to analyse probability of staying in initial non-failure state, $S_{0}$. Simulation is performed with the assumption that parameters $\lambda$ are constant for the analysed system. Several service intervals were simulated, from likely to non-likely. The value of parameter $\mu$ is set to different service intervals.

Configuration of the modelled computer system consists of: microprocessor Intel Core 2 Quad, Kingston RAM 4GB DDR3 $1600 \mathrm{MHz}$, and hard disk Western Digital Velociraptor 1TB. Values of the MTBF are taken from web resources [15 - 17]. Microprocessor's MTBF is equal to 73803 hours (3.37 years), RAM's MTBF is equal to 6618133.7 hours (302.2 years) and HDD's MTBF is equal to 1400000 hours (63.93 years).

\subsection{Simulation results / Rezultati simulacije}

Figure 4 shows that probability of staying in initial state is $50 \%$ after one year. Furthermore, probability of changing the state is $90 \%$ in three year period.

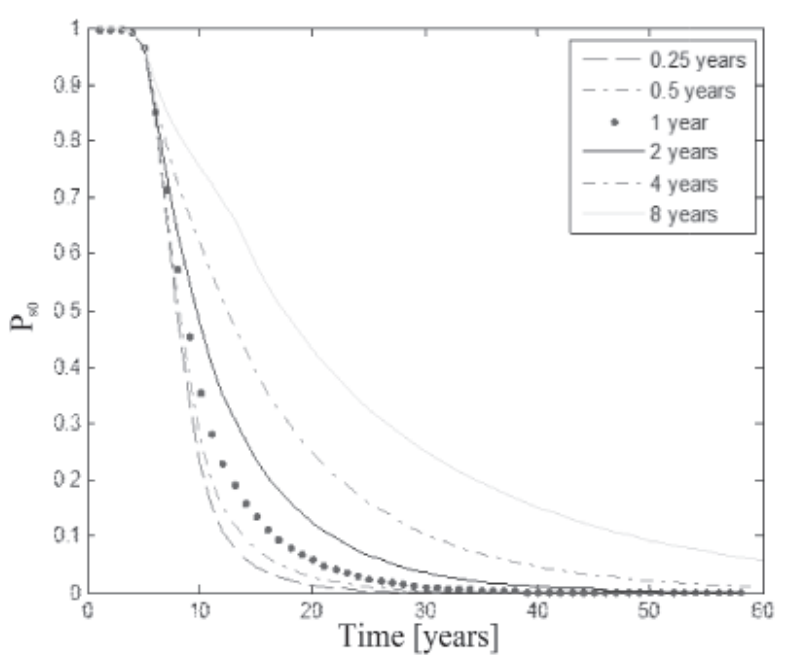

Figure 4 Probability of non-failure state without service and in regular service intervals from 0.25 to 8 years in simulation of reliability

Slika 4. Vjerojatnost stanja bez greške bez servisiranja i tijekom servisiranja u redovitim razmacima od 0,25 do 8 godina pri simuliranju pouzdanosti

Figure 5 shows probability of changing state into failure state, $\mathrm{Pk}$, obtained in simulation of reliability. Simulation of availability implies that probability of staying in the initial state is $50 \%$ in 1.7 years interval for service intensity of $0.5,3$ years for 1 -year service interval and 7.5 years for 2 services per year.

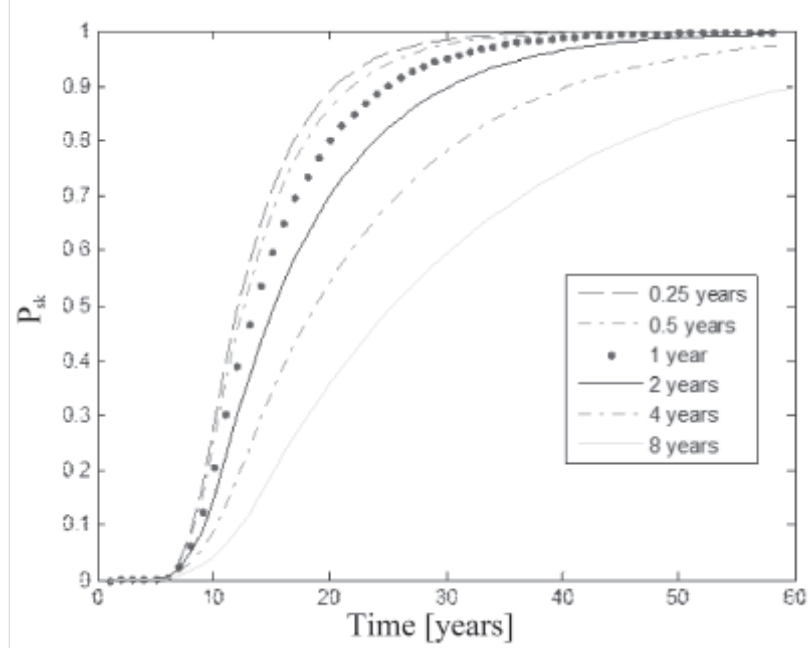

Figure 5 Probability of failure state without service and in regular service intervals from 0.25 to 8 years in simulation of reliability Slika 5. Vjerojatnost stanja greške bez servisiranja i tijekom servisiranja u redovitim razmacima od 0,25 do 8 godina pri simuliranju pouzdanosti

The probability that the system will not stay in the initial state is equal to $90 \%$ for availability simulation and time period depends on repair's intensity. If repair's intensity is 0.5 , then the $90 \%$ is obtained after 12 years. For repair's intensity $1,90 \%$ probability is obtained after 19 years. 33 years is the time to get to $90 \%$ probability in intensity of repairs of factor 2 .

In the second step, the developed simulation model is used to analyse probability of failure state. Figure 6 shows probability of staying in the initial state for availability simulation. Legend data (years) denoted service intervals.

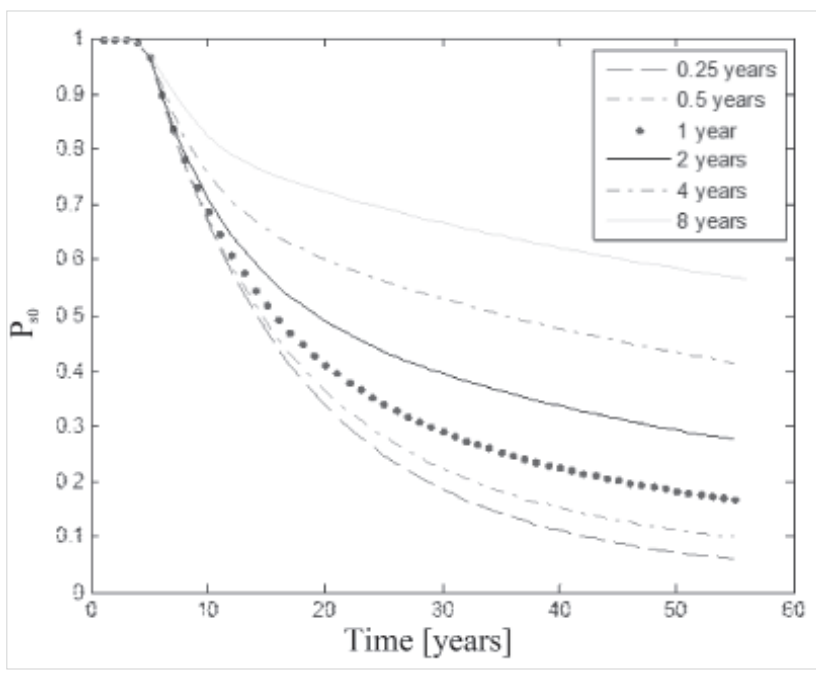

Figure 6 Probability of staying in the initial state for availability simulation in dependence to service intervals

Slika 6. Vjerojatnost ostajanja u početnom stanju pri simuliranju raspoloživosti ovisno o razmacima servisiranja 
Table 5 Total reliabilities/availabilities for all states when system is operational

Tablica 5. Ukupne pouzdanosti/raspoloživosti za sva stanja kad sustav radi

\begin{tabular}{|c|c|c|c|c|c|c|c|c|c|}
\hline $\begin{array}{l}\text { After } \\
\text { years }\end{array}$ & $\begin{array}{c}\text { Service } \\
\text { intervals } \\
\text { (years) }\end{array}$ & Availability & Reliability & $\begin{array}{c}\text { Service } \\
\text { intervals } \\
\text { (years) }\end{array}$ & Availability & Reliability & $\begin{array}{c}\text { Service } \\
\text { intervals } \\
\text { (years) }\end{array}$ & Availability & Reliability \\
\hline 1 & 1 & 0.991 & 0.989 & 2 & 0.9695 & 0.9886 & 0.5 & 0.9969 & 0.9972 \\
\hline 3 & 1 & 0.945 & 0.9213 & 2 & 0.9 & 0.9213 & 0.5 & 0.98 & 0.9773 \\
\hline 5 & 1 & 0.9134 & 0.8294 & 2 & 0.8742 & 0.8295 & 0.5 & 0.9586 & 0.9427 \\
\hline
\end{tabular}

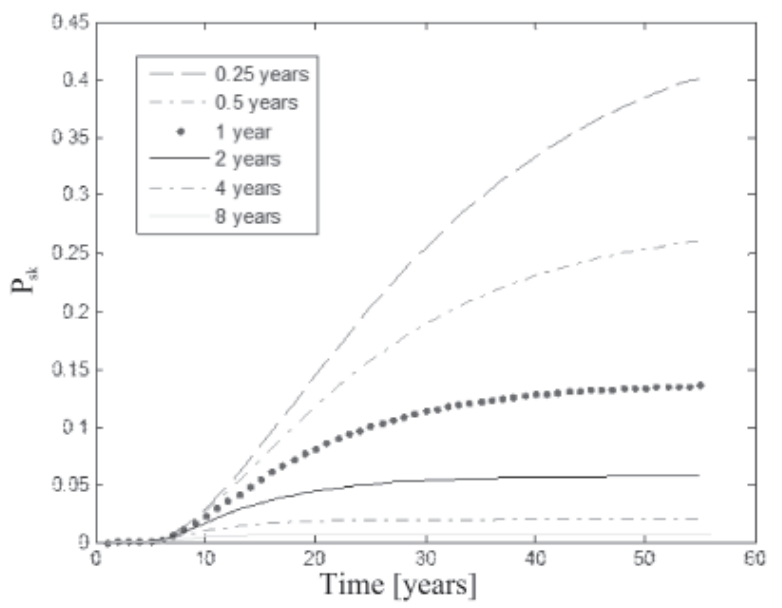

Figure 7 Probability of failure state in availability simulation Slika 7. Vjerojatnost stanja greške pri simuliranju raspoloživosti

Time dependence of transition to state of failure is shown in Figure 7. Legend data (years) denoted service intervals. It can be seen that probability of failure state (in case of reliability) is about $50 \%$ after 4 years, $50 \%$ after 4 years, and $90 \%$ after 10 years. In case of availability, $50 \%$ probability of failure is after 6 years with repair's intensity of $0.5,8$ years with repair's intensity of 1 , and 11.5 years for repair's intensity of factor 2 .

Probability of failure state is $90 \%$ after 17 years with intensity of repairs $0.5,24$ years for repair's intensity of factor 1 , and 37.4 years for repair's intensity of factor 2 (service - 2 times per year).

Table 5 shows results for total reliabilities/availabilities for all states when system is operational in cases of $0.5,1$, and 2 years service intervals after 1,3 , and 5 years of operation.

\section{CONCLUSION / Zaključak}

Many published research works are concerned about physical phenomena behind system failure. We used a different approach: known MTBF data for known components are used to predict reliability and availability of the computer system without introducing physical layer of failure mechanisms. The motive is in companies, which are not interested in physical phenomena research but in knowing how safe their fleet is.

It is shown that computer with one redundancy per component can be operational without repairs for 1 year with $50 \%$ probability.

If we maintain the system and repair it, the system will have longer life expectancy. For more repairs system life is longer. But, if we repair the system all the time, we won't be able to work on it. Therefore, there is no economic value of all-time-repaired systems. Therefore, optimum between system's availability and repair's rate should be found.

An interesting implication is the choice of components used for the simulation. We used "normal" PC components. It would be interesting to compare these data to the corresponding components in the aboard computer systems.

Nowadays trends are to include as much as possible PC computers aboard ships, but it is still limited trend. The most computer-controlled systems use PLCs (Programmable Logic Controller).

Measures to increase reliability of ships' computer systems have economic costs. For example, increase in redundancy means better reliability, but also new computers in standby. However, distributed networks can reduce such cost and increase reliability. Hence, old ships should be modernized, if possible, to increase redundancy by integrated ship's networks.

Similar approach as in this paper can be used if more components are taken into account. In such case, we just add more parallel branches in series.

\section{Acknowledgment / Zahvala}

This work is a part of the research at the project "Functional integration of University of Split, Faculty of Maritime Studies, Faculty of Chemistry and Technology, and Faculty of Science through Development of Scientific and Research Infrastructure in the Building of 3 Faculties, KK.01.1.1.02.0018" financed by EU, performed by scientific group "New technologies in maritime."

\section{REFERENCES / Literatura}

[1] Kuzmanić, I., Vujović, I. (2015). Reliability and Availability of Quality Control Based on Wavelet ComputerVision. London: Springer Verlag. https://doi.org/10.1007/978-3-319-13317-1

[2] Stazić, L., Komar, I., Mihanović, L., Mišura, A. (2018). "Shipowner's Impact on Planned Maintenance System Database Quality Grades Resemblance Equalization". Transactions on Maritime Science, Vol. 7, No. 1, pp. 5-22. DOI: 10.7225/toms.v07.n01.001. https://doi. org/10.7225/toms.v07.n01.001

[3] Guclu, S. O., Ozcelebi, T., Lukkien, J. (2016). “Distributed Fault Detection in Smart Spaces Based on Trust Management". Procedia Computer Science, Vol. 83, pp. 66-73. https://doi. org/10.1016/j.procs.2016.04.100

[4] Srinivasan, J., Adve, S. V., Bose, P., Rivers, J. A. (2004). "The Case for Lifetime ReliabilityAware Microprocessors". Proc. 1st International Symposium on Computer Architecture (ISCA-04), June 2004. Available at: http://rsim.cs.illinois.edu/Pubs/srinivasan_isca04. pdf [accessed 20/2/2018] https://doi.org/10.1109/ISCA.2004.1310781. https://doi. org/10.1145/1028176.1006725

[5] Lala, P. K. (2001). Self-checking and fault-tolerant digital design. San Diego: Academic Press.

[6] Srinivasan, J., Adve, S. V., Bose, P., Rivers, J., Hu, C. K. (2003). RAMP: A Model for Reliability Aware Microprocessor Design, IBM Research Report, RC23048 (W0312-122), 2003. Available at: http:// www.cs.utexas.edu/ hestness/papers/srinivasan-rampdetail.pdf [accessed 16/3/2018]

[7] Shin, J. (2008). Lifetime Reliability Studies for Microprocessor Chip Architecture. Ph. D. thesis, Faculty of the Graduate School University of Southern California. Available at: http:// digitallibrary.usc.edu/cdm/ref/collection/p15799coll127/id/108618 [accessed 15/10/2012]

[8] Rodriguez, J., Remack, K., Gertas, J., Wang, L., Zhou, C., Boku, K., Rodriguez-Latorre, J., Udayakumar, K. R., Summerfelt, S., Moise, T., Kim, D., Groat, J. Eliason, J., Depner, M., Chu F. (2010). "Reliability of Ferroelectric Random Access memory embedded within $130 \mathrm{~nm}$ CMOS". Proc. 2010 IEEE International Reliability Physics Symposium (IRPS), 2-6 May 2010, Anaheim, USA. https://doi.org/10.1109/IRPS.2010.5488738

[9] Kontoleon, J. M., Stergiou, A. (1991). "Reliability analysis and design of a fault-tolerant random access memory system". Microelectronics and Reliability, Vol. 31, pp. 1063-1067. https://doi.org/10.1016/0026-2714(91)90288-I

[10] Li, Q., Patel, U. (2005). “Enabling Memory Reliability, Availability, and Serviceability Features on Dell PowerEdge Servers". Dell Power Solutions, Vol. 8, pp. 1-4. Available at: http://www. dell.com/downloads/global/power/ps3q05-20050176-Patel-OE.pdf [acces sed 13/2/2018]

[11] Louit, D., Pascuall, R., Banjevic, D., Jardine, A. K. S. (2011). “Optimization Models for Critical Spare Parts Inventories - A Reliability Approach". Journal of the Operational Research Society, Vol. 62, No. 6, pp. 992-1004. https://doi.org/10.1057/jors.2010.49

[12] Čoko, M. (2013). Analysis of Reliability and Availability of the Computer System in Matlab. Master's thesis, University of Split, Faculty of Maritime Studies, Split, Croatia.

[13] Young, R. B. (2003). Reliability Transform Method. Master's thesis, Virginia Polytechnic Institute and State University, USA.

[14] Faulin, J., Juan, A. A., Martorell, S., Márquez, J. E. R. (2010). Simulation Methods for Reliability and Availability of Complex Systems, London: Springer-Verlag. https://doi. org/10.1007/978-1-84882-213-9

[15] Microprocessor failure intensity, Data Sheet Archive. Available at: http://www. datasheetarchive.com/processor+mtbf+pentium+4-datasheet.html [accessed 12/2/2019]

[16] RAM failure rate, Alion Science. Available at: http://src.alionscience.com/cgi-src/netforum/ reliability_forum/a/8--1.377.1.73.0 [accessed 12/2/2019]

[17] HDD failure rate, WDC Corporation. Available at: http://www.wdc.com/wdproducts/ library/OVR/ENG/2178-001010.pdf [accessed 12/2/2019] 